\title{
Capsule commentary on Lewis Hunter et al., Factors Influencing Hospital Admission of Non-Critically III Patients Presenting to the Emergency Department: A Cross-Sectional Study
}

\author{
Kathi Glauner, M.D. Ph.D. \\ University of Kansas Hospital, Kansas, KS, USA. \\ J Gen Intern Med 31(1):101 \\ DOI: $10.1007 / \mathrm{s} 11606-015-3448-6$ \\ (c) Society of General Internal Medicine 2015
}

$\mathrm{T}$ his single-site cross-sectional study by Lewis Hunter et al. ${ }^{1}$ addresses variation in admission rates for patients with similar clinical presentations who are evaluated in the emergency department (ED). The authors question whether non-clinical factors might explain this variability. Via survey responses, physicians reported the influence of medical acuity and non-clinical factors on their decisions to admit. Investigators found that greater than $90 \%$ of admissions were influenced by non-clinical factors, with over half being strongly or moderately influenced. While this did not likely change the disposition of high-acuity patients, it may have been enough to provoke admission of some low-risk patients.

The authors wonder if this information could be used in the development of resources for emergency department patients that would allow safe discharge from the ED and assurance of follow-up testing. Decision rules with specific admission vs. observation vs. outpatient follow-up guidelines already exist for conditions such as syncope, chest pain, and TIA. Readily available follow-up for patients who are suitable for outpatient treatment could decrease the number of patients admitted based on concerns about follow-up or lack of rapid diagnostic testing. Syncope or observation units run by emergency departments could also help to reduce hospital admissions ${ }^{2,3}$.

While the study is difficult to generalize due to its short duration, single location, and lack of nighttime data points, the information is timely. Hospitals and physicians are being

encouraged to bring down the cost of admissions and the overall cost of medical care to patients. Rather than cutting costs on admitted patients, this study suggests that reducing admissions by providing alternatives that address physician concerns could be a viable option. Prior investigations of the efficacy of case management and social workers in reducing admissions have been promising ${ }^{4}$. Perhaps the additional information provided by this study will encourage further progress in reducing the number of admissions of patients who could be followed up as outpatients.

Conflict of Interest: The author has no conflicts of interest with regard to this article.

Corresponding Author: Kathi Glauner, M.D. Ph.D.; University of Kansas Hospital, Kansas, KS 66160, USA (e-mail: kglauner@kumc.edu).

\section{REFERENCES}

1. Lewis Hunter AE, Spatz ES, Bernstein SL, Rosenthal MS. Factors Influencing Hospital Admission of Non-critically Ill Patients Presenting to the Emergency Department: A Cross-sectional Study. Journal of General Internal Medicine. 2015 doi:10.1007/s11606-015-3438-8.

2. Ungar A, Tesi F, Chisciotti VM, Pepe G, Vanni S, Grifoni S, Balzi D, Rafanelli M, Marchionni N, Brignole M. Assessment of a structured management pathway for patients referred to the Emergency Department for syncope: results in a tertiary hospital. Europace. 2015 May 14. Pii; euv 106

3. Sun BC, McCreath H, Liang LJ, Bohan S, Baugh C, Ragsdale L, et al. Randomized clinical trial of an emergency department observation syncope protocol versus routine inpatient admission. Annals of Emergency Medicine. 2014;64:167-75.

4. Bristow DP, Herrick CA. Emergency Department Case Management: The Dyad Team of Nurse Case Manager and Social Worker Improve Discharge planning and Patient and Staff Satisfactio While Decreasing Inappropriate Admissions an Costs: A Literature Review. Lippincott's Case Management. 2002;7(6):243-251. 